\title{
A passive wireless tracking system for indoor assistive monitoring
}

Yau Hee Kho, Nguan Soon Chong, Refik Caglar Kizilirmak

- School of Engineering

Abstract

This paper presents a design concept and implementation of an indoor passive tracking system that utilises an array of Wi-Fi transceivers, and without any electronic device or tag attached to the object being tracked. Such tracking is made possible by exploiting the fundamental characteristic of signal attenuation due to object blocking, i.e. shadowing, that is prevalent in a typical wireless communication system. By detecting significant signal attenuation in the system (i.e. by measuring the received signal strength value), it is possible to infer that an object is blocking the line-of-sight (LOS) link in a transceiver set and therefore transforming the existing hardware configuration into a proximity sensors network.

Original language

Title of host publication

Publisher

Pages

Number of pages

Volume

ISBN (Print)

State

Event

Other

Country

City

Period
English

Proceedings of the International Conference on Sensing Technology, ICST

IEEE Computer Society

$138-142$

5

2016-March

9781479963140

Published - Mar 212016

9th International Conference on Sensing Technology, ICST 2015 -

Auckland, New Zealand

9th International Conference on Sensing Technology, ICST 2015

New Zealand

Auckland

$12 / 8 / 15 \rightarrow 12 / 11 / 15$ 
Kho, Y. H., Chong, N. S., \& Kizilirmak, R. C. (2016). A passive wireless tracking system for indoor assistive monitoring. In Proceedings of the International Conference on Sensing Technology, ICST. (Vol. 2016March, pp. 138-142). [7438379] IEEE Computer Society. DOI: 10.1109/ICSensT.2015.7438379 